\title{
Carga Mental e Ergonomia
}

\author{
Fábio de Paula Corrêa \\ Orientador: Prof. Dr. Roberto Moraes Cruz \\ Dissertação de Mestrado apresentada no Programa de Pós Graduação em Engenharia de \\ Produção da Universidade Federal de Santa Catarina.
}

Este estudo investiga a utilidade e a possibilidade de uso da base teórica de Carga Mental no trabalho, em uma situação de trabalho específica no que se refere aos seus aspectos ergonômicos. Procura-se indicar as vantagens e desvantagens decorrentes da incorporação e utilização dos conceitos e métodos relacionados à Carga Mental de Trabalho. A revisão teórica buscou referenciar o estado da arte da Carga Mental de Trabalho, recorrendo a uma literatura pouco conhecida pelos ergonomistas. Nesta revisão caracterizam-se as diferentes abordagens teóricas sobre o conceito de Carga Mental, suas limitações e sua multidimensionalidade. Confrontamos os posicionamentos de pesquisadores da Ergonomia sobre a utilização da teoria de carga de trabalho em um contexto ergonômico, onde se constata um conflito de posições não existindo consenso. Foram comparados também conceitos e procedimentos da Ergonomia com métodos utilizados nos estudos de Carga Mental. A noção de Regulação, usada em Ergonomia é contraposta à noção de Desempenho utilizada nos estudos de Carga Mental. Na pesquisa de campo utiliza-se um dos métodos mais consagrados para acesso à Carga Mental de Trabalho (NASA-TLX), em uma situação real de trabalho com forte característica mental (Call Center em uma empresa de Telecomunicações).

O estudo de campo foi feito a partir de trinta funcionários, sendo que a aplicação do instrumento NASA-TLX foi acompanhada de entrevistas e questionários para levantamento de diversas variáveis incidentes na situação de trabalho. A população inicial foi subdividida em dois subgrupos cujo conteúdo do trabalho tinha diferenças significativas. Os resultados mostraram que a média da carga mental de cada subgrupo reflete a influência do conteúdo do trabalho sobre as escalas do instrumento. As entrevistas permitiram a associação de aspectos pessoais e qualitativos aos resultados de carga mental. A comparação dos resultados individuais de dois sujeitos, cada um pertencente a um dos subgrupos, mostrou que os resultados apresentam coerência mesmo ao nível individual. As variáveis coletadas através de questionário, se mostraram sensíveis à regressão linear feita em relação à sobrecarga. Assim, por exemplo, nota-se que a sobrecarga é menor para os operadores mais idosos aumentando significativamente para os mais jovens. Já a sobrecarga é diretamente proporcional para variáveis tais como, queixas em relação a organização, sintomas dolorosos, queixas em relação ao ambiente de trabalho.

O que se pode concluir das aplicações em campo é que os métodos de acesso à Carga Mental permitem ao ergonomista chegar a conclusões rápidas e precisas acerca dos problemas ergonômicos nesta área. O que observamos é que, mesmo pesquisando uma 
população pequena em relação ao universo de trabalhadores na atividade, pode-se descobrir uma infinidade de fatores que geram Carga Mental na situação de trabalho. Fica evidente a necessidade de associação dos métodos de acesso, a outros recursos como questionário e entrevista, uma vez que o resultado de Carga, puro e simples não é muito útil do ponto de vista ergonômico.

A possibilidade de realizar regressão de variáveis com a sobrecarga enriquece sobremaneira a análise. Por exemplo, ao verificarmos que a sobrecarga é inversamente proporcional à idade, ocorreu uma confirmação de suspeitas de inadequação do perfil da população à atividade. Estas suspeitas haviam surgido durante a execução de uma análise ergonômica anterior a nossa pesquisa, conduzida no mesmo local e em trabalho realizados em outros contextos similares.

Este resultado é importante de diversas maneiras: primeiro no sentido em que superamos a idéia de que os mais idosos estariam sujeitos a maior sobrecarga ao lidar com equipamentos de informática. Ao utilizarmos uma base teórica como a Carga Mental, vemos que isto não é totalmente verdadeiro. Isto ocorre, por que a análise de carga supera a interface tecnológica, considerando a influência de fatores sociais em sua constituição. Em uma Análise Ergonômica tradicional, muito provavelmente, estaríamos preocupados com a interface entre o operador e o sistema de informação, tendo dificuldades para operar em outros domínios. A base conceitual de carga desvenda a influência de variáveis incidentes na situação de trabalho.

Outro fator interessante é que mesmo trabalhando com uma população pequena e utilizando um recurso estatístico simples como a média aritmética, é possível observar a influência do conteúdo das atividades nos resultados de carga mental. Na comparação entre os dois grupos fica nítida a diferença qualitativa na constituição da Carga Mental de cada um deles.

Salienta-se também que, de modo geral, os resultados que encontrados em campo tem paralelo com as pesquisas de Xie e Salvendy (2000) nos seguintes aspectos: a importância do fator relacionado à pressão de tempo como gerador de sobrecarga observado tanto no aspecto qualitativo como no quantitativo e a importância da flexibilidade organizacional como fator redutor de sobrecarga.

Um aspecto menos interessante é que efetivamente a aplicação destes métodos, ainda que acompanhada de outros instrumentos, não permite conclusões imediatas sobre a questão da regulação da Carga. Observamos que o aspecto de regulação, considerando ou não o conceito de carga é por demais complexo para ser equacionado em um levantamento rápido. Hart (1999), como já vimos, propõe a associação do conceito de carga ao de desempenho, servindo este de parâmetro para avaliar o modo como o indivíduo lida com a própria carga. Este aspecto foge ao desenho de nosso estudo, sendo então impossível sermos conclusivos a este respeito.

Finalmente, falando da experiência deste autor na utilização dos métodos em campo, pode-se dizer que a utilização inicial destes métodos em trabalhos com forte componente mental é de grande valia para a Análise Ergonômica, podendo servir de guia a levantamentos adicionais em torno de questões onde é necessário maior esclarecimento. 
Do ponto de vista da fundamentação teórica, vemos algumas vantagens na adoção do conceito de Carga de Trabalho que não devem ser desprezadas. Imaginamos que com os métodos de acesso à Carga que existem hoje, se possa fazer uma gestão da Carga, tanto física como mental, nos locais de trabalho. Como já havíamos mencionado, Hart (1999) propõe um programa de Gerenciamento da Carga Mental em trabalhadores da aviação civil e militar. Esta possibilidade torna-se muito difícil no modelo ergonômico tradicional, pois não existiria uma maneira prática do ergonomista acompanhar a evolução ou retrocesso do assunto.

Observamos que o conceito de Carga Mental tem amplas possibilidades de interação com a Ergonomia Cognitiva, especialmente quando falamos de Arquiteturas Cognitivas e modelos mentais em sistemas complexos. Associamos por exemplo uma maior ou menor carga conforme o tipo de raciocínio, quando utilizamos uma arquitetura como a de Richard, ou investigamos em que etapa ou etapas do processo decisório existe a maior carga, quando utilizamos a arquitetura de Rassmussem.

Notamos também que a Carga Mental apresenta um arcabouço teórico que permite ao pesquisador transitar entre aspectos micro e macro do trabalho com muita facilidade e sempre dentro de uma mesma base referencial. Podemos tanto analisar a carga mental do indivíduo, como podemos analisar a carga mental de um determinado grupo de trabalhadores. Isto ficou bem evidenciado em nosso trabalho de campo, pois os resultados foram significativos tanto ao comparar a carga mental de dois sujeitos, como quando comparamos a carga mental dos dois subgrupos populacionais através da carga média.

Discordamos de Guérin, Laville, Daniellou, Duraffourg e Kerguelen (1997) quando estes dizem que a noção de Carga de Trabalho é estéril. Pensamos justamente o contrário, na medida em que a noção de carga permite o debate com a empresa. Este debate pode ser conduzido tanto sob aspectos quantitativos da carga como em seus aspectos qualitativos. Nos nossos levantamentos de campo, por exemplo, verificamos uma série de fatores de frustração na população trabalhadora passíveis de quantificação e mais importante: passíveis de identificação de suas origens. Mas esta quantificação não será estéril, na medida em que é feita sempre em relação a alguma outra grandeza. Assim se uma população tem sua carga mental mais associada a um fator como frustração ao invés de exigência mental, isso já direciona a intervenção na situação de trabalho.

Os empregadores têm condições então de acompanhar as transformações na Carga tanto nos aspectos qualitativos como quantitativos. Os profissionais de outras áreas de conhecimento tem uma base teórica a ser considerada quando projetam seus sistemas de trabalho. A partir do conceito de Carga de trabalho podem-se realizar discussões acerca de pausas, divisão do trabalho, remuneração, organização das decisões, e muitos outros aspectos do trabalho.

Entende-se que novos campos de pesquisa se apresentam aos ergonomistas, especialmente na interação entre as arquiteturas cognitivas e aspectos da carga mental. Pode-se pesquisar, por exemplo, que tipo de raciocínios produzem mais carga mental em dada situação de trabalho. Outro campo de interesse é aquele que associa aspectos da saúde à carga mental de trabalho. Pode-se, por exemplo, tentar quantificar qual a influência da 
carga mental no desenvolvimento das DORT (Doença ósteo-articular relacionada ao trabalho).

Referências:

BORG, G. Psychophysical scaling with applications in physical work and the perception of exertion. Scandinavian Journal of Work Environmental Health, vol 16 (suppl. 1): 55$58,1990$.

CRUZ, R.M. Psicodiagnóstico de Síndromes Dolorosas Crônicas Relacionadas ao Trabalho. Florianópolis, 2001. Tese (Doutorado em Engenharia de Produção) - Programa de Pós-Graduação em Engenharia de Produção . Universidade Federal de Santa Catarina. 2001.

COLLE, H., REID, G.B. Context effects in subjective mental workload ratings. Human Factors. Vol 40 (4), dez./98, p. 591-600.

CASALI, J. G. \& WIERWILLE, W. W. A comparison of rating scale, secondary-task, physiological, and primary-task workload estimation techniques in a simulated flight task emphasizing communications load. Human Factors, vol 25, 623-641, 1983.

GUÉRIN,F.; LAVILLE, A.; DANIELlOU F.; DURAFFOURG, J.; KERGUELLEN, A.; Compreender o Trabalho para Transformá-lo. 2001. Ed. Edgard Blücher.

HART. Gerenciamento da Carga de Trabalho da Tripulação: um fator crítico no desempenho do sistema. Revista SIPAER, Maio 1999.

HENDY, K.C. et al de 1993 publicado na revista HUMAN FACTORS vol. 35.

ISO 10075. Ergonomic Principles Related to Mental Work-Load - General Terms and Definitions. 1991.

ISO 10075-2. Ergonomic Principles Related to Mental Workload - Part 2: Design principles. 1996

LAUrell, A. , NORIEGA, M. Processo de Produção e Saúde. São Paulo: Hucitec, 1989.

LEPLAT, J., CUNY, X. Introdução à psicologia do trabalho. Trad. Helena Domingos. Lisboa: Fundação Calouste Gulbenkian, 1983.

MACDONALD, W.; BENDAK, S. Effects of Workload Level and 8- versus 12-h Workday Duration on test Battery Performance et al de 2000 publicado no International Journal of Industrial Ergonomics vol. 26.

MANUAL DO NASA TLX - NASA Ames Research. Califórnia, EUA: 1986. 
MIYAKE, S. Multivariate Workload Evaluation Combining Physiological and Subjective Measures, 2001. International Journal of Psychophysiology 40, p. 233-238

MORAY, N. Mental Workload Since 1979, International Reviews of Ergonomics, 2, p. 123-150, 1988.

MONTMOLLIN, M. de. Vocabulaire de L’ Ergonomie. Paris: Octares (p.), 1995.

MESHKATI, N.; HANCOCK, P.A.; RAHIMI, M. Techiniques in Mental Workload Assessment. P. $605-6271990$.

OGDEN, G.; LEVINE, J. \& EISNER, E., Measurement of Workload by secondary tasks. Human Factors, 21, 529-548, 1979.

O`BRYAN , S. J.; MACDONALD W.A.; EVANS, O.M. A Comparison of Some Workload Analysis Techniques. Centre for Ergonomics and Human Factors, La Trobe University.

RASSMUSSEN, J. Modèls en Analyse du Travail. Viége:Mandarga, 1991.

SANTOS, N. dos, FIALHO, F. Manual de Análise Ergonômica do Trabalho. Curitiba: Gênesis, 1997.

Richard, J. F. Les Activités Mentales. Paris: Armand Polin, 1990.

SPERANDIO, J. C. L'ergonomie du travail mental. Paris: Masson, 1988.

WISNER, A. A inteligência no trabalho: Textos selecionados de ergonomia. São Paulo: Fundacentro, 1997.

XIE, B.; SALVENDY, G. Prediction of Mental Workload in Single and Multiple Tasks Environments, 2000. International Journal of Cognitive Ergonomics p. 213-242.

Aprovada por:

Prof. Edson Pacheco Paladinni, Dr.

Coordenador do Programa - Universidade Federal de Santa Catarina

Prof ${ }^{\mathrm{a}}$. Ana Regina de A. Dutra, Dr ${ }^{\mathrm{a}}$.

Universidade Federal de Santa Catarina

Prof. Emil Kupek, Dr.

Universidade Federal de Santa Catarina 


\begin{abstract}
The present study looks into the utility and the possibility of using a theoretical basis of Work Mental Load in a specific work situation. It intends to show the advantages resulted from the incorporation and utilization of the concepts and methods related to the Work Mental Load. The theoretical review tried to refer the current Work Mental Load status, looking into a literature usually unknown by the ergonomists. This review is characterized by its variable theoretical approaching over the concept of Mental Load, its limitations and multidimensionality. We have confronted the point of view of Ergonomics researchers concerning the utilization of the mental load theory at an ergonomic context, although it is clear that there is still a conflict in their opinions, being difficult to reach a consensus. This study has also compared the concepts and proceedings of the Ergonomics with methods used on Mental Load studies. The idea of Regulation used in Ergonomics is confronted to the idea of Performance used in Mental Load research. At the field research it has been used one of the most reliable methods for Mental Load investigation (NASA-TLX) in a real situation with a strong mental characteristic (Call Center of a Telecommunications company). The results suggest numberless advantages to the ergonomist when adopting this approaching since it allows to quantify and to qualify the mental load, analyzing it into several dimensions of the work situation and reporting this theoretical basis to a group of variables incident in a work situation.
\end{abstract}

Key Words: Workload, Mental Workload, Ergonomics, NASA-TLX, Call Center. 